\title{
Microarray Analysis And Functional Prediction of Differentially-Expressed Circular RNAs In Acquired Middle Ear Choleateatoma
}

\section{Shumin Xie}

Xiangya Hospital Central South University

Li Jin

Second Xiangya Hospital

Tuanfang Yin

Second Xiangya Hospital

Jihao Ren

Second Xiangya Hospital

Wei Liu ( $\sim$ liuwei007@csu.edu.cn )

CSU: Central South University https://orcid.org/0000-0002-2798-7664

\section{Research}

Keywords: circRNA, cholesteatoma, etiopathogenesis, ceRNA, microarray analysis

Posted Date: October 5th, 2021

DOl: https://doi.org/10.21203/rs.3.rs-944677/v1

License: (9) This work is licensed under a Creative Commons Attribution 4.0 International License. Read Full License 


\section{Abstract}

Background: Middle ear cholesteatoma is characterized by hyper-proliferation of keratinocytes. Circular RNA (circRNA) plays an essential role in the pathogenesis of many proliferative diseases. However, the role of circRNA in the etiopathogenesis of middle ear cholesteatoma is rarely investigated so far. Our aim was to investigate the differential expression profiling of circRNAs between acquired middle ear cholesteatoma and normal skin, and to indentify potential circRNAs contributing to the etiopathogenesis of middle ear cholesteatoma. Microarray analysis and functional prediction were performed to investigate the circRNA expression profiling between middle ear cholesteatoma and normal skin. Validation of differentially expressed circRNAs was conducted by qRT-PCR. Prediction of $\mathrm{m}^{6} \mathrm{~A}$ modification was also carried out.

Results: Microarray analysis displayed that totally 93 up-regulated and 85 down-regulated circRNAs were identified in middle ear cholesteatoma. Through validation, expressions of hsa_circRNA_104327 and hsa_circRNA_404655 were significantly higher, while hsa_circRNA_000319 was significantly downregulated in cholesteatoma. GO classification, KEGG pathway, and ceRNA network analyses suggested that these differentially expressed circRNAs might play importantant roles in the etiopathogenesis of middle ear cholesteatoma. Prediction of $\mathrm{m}^{6} \mathrm{~A}$ modification exhibited that hsa_circRNA_000319 possessed $4 \mathrm{~m}^{6} \mathrm{~A}$ sites with very high confidence, and hsa_circRNA_404655 had $3 \mathrm{~m}^{6} \mathrm{~A}$ sites with high confidence.

Conclusions: Our study indicated possible roles of differentially expressed circRNAs in the etiopathogenesis of middle ear cholesteatoma. Targeting on these differentially expressed circRNAs may provide a new and effective treatment for middle ear cholesteatoma in the future.

\section{Background}

Accompanied with chronic otitis media, middle ear cholesteatoma is a locally destructive squamous epithelial lesion with clinical features such as hyperproliferation, migration, aggressiveness, and recurrence. Although middle ear choleateatoma is pathological benign, it progressly develops and leads to erosion of adjacent bony structures, resulting in vestibular dysfuction, labyrinthine fistulae, facial paralysis, hearing loss and serious intracrnial complications $[1,2]$. The incidence of middle ear cholesteatoma has been reported as high as 9.2 per 100,000 populations in Europe, while even higher in Asians [3]. Although a wide spectrum of hypotheses have been put forward, the exact etiopathogenesis of middle ear cholesteatoma is not clear yet. Since there is no effective drug for treating middle ear cholesteatoma, surgical resection is still the only option presently. However, surgical resection is often accompanied by dysfunction of middle and inner ear, which may cause further hearing loss [4]. Due to its recurrence, a few of patients have to receive repeated surgeries. Meanwhile, for patients with poor general conditions, such as severe respiratory diseases, cardiovascular and cerebrovascular diseases, etc., cholesteatoma resection cannot be performed. 
Circular RNA (circRNA) is a special endogenous non-coding RNA with a unique circular structure, derived from exons or introns [5]. MicroRNA (miRNA) regulates the translation of message RNA (mRNA) at the post-transcriptional level. Through binding their targeted messenger RNA (mRNA) at the 3'-untranslated region, miRNA initiates degradation of mRNAs [6]. CircRNA is rich in miRNA response element (MRE), and functions as efficient miRNA sponges. Act as a competing endogenous RNA (ceRNA), circRNA is able to suppress the activity of miRNA, release miRNA's inhibition on its targeted mRNA, and up-regulate the expression of target gene, through binding to miRNA [7]. CircRNA is widely present in mammalian cells and plays an important role in a series of life processes such as cell proliferation, differentiation, apoptosis, migration and body metabolism [8].

CircRNA plays an essential role in the pathogenesis of many proliferative diseases, such as hyperproliferation of keratinocytes in psoriasis [9], hypoxia-induced proliferation of vascular endothelial cells [10], skeletal myoblast proliferation [11], and so on. Currently, it is recognized internationally that middle ear cholesteatoma is characterized by hyper-proliferation of keratinocytes. A variety of epithelial cell proliferation markers such as proliferating cell nuclear antigen (PCNA), Ki-67, cytokeratin (CK) 13, and CK16 have been demonstrated to express significantly high in middle ear cholesteatoma epithelium [1214]. However, the role of circRNA in the etiopathogenesis of middle ear cholesteatoma is rarely investigated so far.

In our research, we investigated the differential expression profiling of circRNAs between acquired middle ear cholesteatoma and normal skin by microarray technology. Expression of several selected circRNAs was validated through quantitative real-time polymerase chain reaction (qRT-PCR), purposing to recognize meaningful circRNAs contributing to the pathogenesis of middle ear cholesteatoma.

\section{Results}

\section{Differential expression of circRNAs in cholesteatoma and normal skin}

In our study, a total of 13,562 expressed circRNAs were detected through microarray analysis. Compared with normal skin, totally 93 up-regulated (hsa_circRNA_104327, hsa_circRNA_404655, etc.) and 85 downregulated (hsa_circRNA_000319, hsa_circRNA_048764, etc.) circRNAs were identified in middle ear cholesteatoma tissues with a value of $F C \geq 2(P<0.05)$. The differentially expressed circRNAs were displayed in a volcano plot (Figure 1A), and then subjected to a scatter plot (Figure 1B) and hierarchical clustering analysis (Figure 1C), to display the variation and differentiation between middle ear cholesteatoma and normal kin. Moreover, among these 178 significantly differentially expressed circRNAs, a total of 145 were exonic. Discoveries in our study indicated that these differentially expressed circRNAs might contribute to the pathogenesis of middle ear cholesteatoma.

\section{Validation for differentially expressed circRNAs}


To validate the circRNAs microarray result, a total of 8 differentially expressed circRNAs were selected for qRT-PCR analysis. In detail, 5 up-regulated circRNAs (hsa_circRNA_103670, hsa_circRNA_048764, hsa_circRNA_404864, hsa_circRNA_104327, hsa_circRNA_404655), and 3 down-regulated circRNAs (hsa_circRNA_101965, hsa_circRNA_000319, hsa_circRNA_100927) were validated. The validation result was accordance with the microarray discoveries partly: compared with normal skin, expressions of hsa_circRNA_104327 and hsa_circRNA_404655 were significantly higher in middle ear cholesteatoma $(P<0.05)$, while the expression of hsa_circRNA_000319 was significantly decreased $(P<0.05)$ (Figure 2$)$.

\section{GO and KEGG}

To preliminarily investigate the function of differentially expressed circRNAs in middle ear cholesteatoma, GO classification and KEGG pathway analysis were carried out on presumptive target genes of validated circRNAs (hsa_circRNA_104327, hsa_circRNA_404655, and hsa_circRNA_000319). Three domains including BP, CC, and MF in GO classification were investigated. In this research, a total of $205 \mathrm{BP}, 28 \mathrm{CC}$, and $28 \mathrm{MF}$ domains were discovered to be significantly enriched $(P<0.05)$. The top-10 enriched $B P, C C$, and MF domains in GO classification were displayed in Figure 3. Meanwhile, regarding on KEGG pathway analysis, 11 signaling pathways in total were demonstrated to be enriched (Figure 4).

\section{ceRNA network analysis}

Based on the ceRNA hypothesis, circRNAs can crosstalk by competing for common miRNAs, with MRE as the foundation of this interaction. In our study, 3 validated significantly differentially expressed circRNAs including hsa_circRNA_000319, hsa_circRNA_104327, and hsa_circRNA_404655 were selected to perform a circRNA-miRNA-mRNA ceRNA network analysis.

The ceRNA network was shown to be composed of 3 circRNAs, 309 miRNAs, and 131 mRNAs (Supplementary Figure 1). We discovered that hsa_circRNA_000319 interacted with hsa-miRNA-4436b-5p, hsa_circRNA_104327 interacted with miRNA-152-5p, and hsa_circRNA_404655 interacted with miRNA3664-3p, with a complementarity based on 7mer-m8, 6mer, offset $6 \mathrm{mer}$, or 8 mer matching types (Figure 5). Moreover, in our previous study [15], compared with normal skin, the expression of miRNA-4436b-5p was demonstrated to be significantly up-regulated in middle ear cholesteatoma ( $F C>2, P<0.05)$, while miRNA-152-5p and miRNA-3664-3p were obviously down-regulated $(F C<0.5, P<0.05)$, which were synchronous with expressions of hsa_circRNA_000319, hsa_circRNA_104327, and hsa_circRNA_404655 in middle ear cholesteatoma. Additionally, several targeted mRNAs for miRNA-3664-3p were discovered, including IQ motif-containing GTPase activating protein 3 (IQGAP3), receptor tyrosine kinase-like orphan receptor 2 (ROR2), Ephrin Type-B Receptor 1 (EPHB1), nucleophosmin 1 (NPM1), chromosome condensation 2 (RCC2), nuclear receptor coactivator 5 (NCOA5), and so on.

\section{Prediction of $\mathrm{m}^{6} \mathrm{~A}$ on differentially expressed circRNAs}


m6A is a post-transcriptional methylation modification that widely presents at the adenosine bases of RNA transcripts. This modification has been suggested to be involved in the regulation of the degradation, subcellular localization, splicing and local conformation changes of the RNA transcripts. In our study, we discovered that hsa_circRNA_000319 possessed $4 \mathrm{m6A}$ sites with very high confidence in total, and hsa_circRNA_404655 had 3 m6A sites with high confidence, while no m6A site with very high or high confidence was found for hsa_circRNA_104327. Details were displayed in Table 1 and Figure 6.

Table 1

Prediction of $\mathrm{m}^{6} \mathrm{~A}$ on differentially expressed circRNAs

\begin{tabular}{|lllll|}
\hline circRNAs & Position & Sequence context & $\begin{array}{l}\text { Combined } \\
\text { score }\end{array}$ & $\begin{array}{l}\text { Decision of } \mathbf{m}^{\mathbf{6}} \mathbf{A} \\
\text { site }\end{array}$ \\
\hline hsa_circRNA_000319 & 365 & $\begin{array}{l}\text { UGAAA GGACC } \\
\text { UGCUC }\end{array}$ & 0.845 & $\begin{array}{l}\text { very high } \\
\text { confidence }\end{array}$ \\
\hline hsa_circRNA_000319 & 418 & $\begin{array}{l}\text { GAUAU UGACA } \\
\text { UUCAU }\end{array}$ & 0.831 & $\begin{array}{l}\text { very high } \\
\text { confidence }\end{array}$ \\
\hline hsa_circRNA_000319 & 511 & $\begin{array}{l}\text { GAAAU UGACU } \\
\text { UGAAU }\end{array}$ & 0.817 & $\begin{array}{l}\text { very high } \\
\text { confidence }\end{array}$ \\
\hline hsa_circRNA_000319 & 1147 & $\begin{array}{l}\text { AGUAU GAACA } \\
\text { UUGAG }\end{array}$ & 0.730 & $\begin{array}{l}\text { very high } \\
\text { confidence }\end{array}$ \\
\hline hsa_circRNA_404655 & 692 & $\begin{array}{l}\text { GGCAC AGACU } \\
\text { CUGGA }\end{array}$ & 0.668 & High confidence \\
\hline hsa_circRNA_404655 & 797 & $\begin{array}{l}\text { AAGUU GAACU } \\
\text { GCAAU }\end{array}$ & 0.619 & High confidence \\
\hline hsa_circRNA_404655 & 1068 & $\begin{array}{l}\text { ACUAC AGACU } \\
\text { UAGUG }\end{array}$ & 0.618 & High confidence \\
\hline
\end{tabular}

\section{Discussion}

CircRNA is a special endogenous non-coding RNA with a unique ring structure, which regulates gene expression at the post-transcriptional level and plays an important role in a series of life processes, such as cell proliferation, differentiation, apoptosis, migration and metabolism. However, there are few studies on circRNAs in middle ear cholesteatoma, and the role of circRNAs in the etiopathogenesis of middle ear cholesteatoma is rarely investigated so far. We totally identified 93 up-regulated and 85 down-regulated circRNAs in cholesteatoma with statistical significance compared with normal skin. Moreover, qRT-PCR was performed to verify the microarray discoveries by detecting the expression of 8 selected circRNAs, including 5 up-regulated circRNAs (hsa_circRNA_103670, hsa_circRNA_048764, hsa_circRNA_404864, hsa_circRNA_104327, hsa_circRNA_404655), and 3 down-regulated circRNAs (hsa_circRNA_101965, hsa_circRNA_000319, hsa_circRNA_100927). Expressions of hsa_circRNA_104327, hsa_circRNA_404655 and hsa_circRNA_00031) were in line with the microarray results, suggesting that these three circRNAs may play a pivotal role in the pathogenesis of middle ear cholesteatoma. The difference between 
microarray results and qRT-PCR validation minght be casue by the false positive in microarray analysis. Furthermore, a large sample size will be enrolled in our following research.

CircRNA is closely related to the pathogenesis of a variety of proliferative diseases, such as excessive proliferation of psoriatic keratinocytes [9], hypoxia-induced vascular endothelial cell proliferation [10], skeletal myoblast proliferation[11] and so on. In the process of these proliferative diseases, circRNA regulates the expression of related target genes through miRNA "sponge" and participates in the etiopathogenesis of these diseases. Wang et al [11]. found that circTitin activated insulin-like growth factor 2 (IGF2)/phosphatidylinositol 3-kinase (PI3K)/Akt signal pathway through adsorption of miRNA432 , which lead to the proliferation and differentiation of skeletal myoblasts. In addition, circRNA is also closely related to tumorigenesis. Studies have found that the expression of circRNA was abnormal in many kinds of tumor tissues, including breast cancer [16], liver cancer [17] and esophageal cancer [18], which was closely related to tumor cell hyperproliferation, invasion and metastasis. A study found that the expression of circSMARCA5 was significantly down-regulated in hepatocellular carcinoma, and was closely related to the hyperproliferation, invasion and metastasis of hepatocellular carcinoma cells. Transfection of circSMARCA5 into the hepatocellular carcinoma cells can up-regulate the expression of target gene tissue inhibitor of matrix metalloproteinase 3 (TIMP3) through adsorption of miRNA-17-3p and miRNA-181b-5p, and ultimately inhibit the proliferation, invasion and metastasis of hepatocellular carcinoma cells [17]. Respecting to middle ear cholesteatoma, recent studies have found that hsa_circRNA_102747 acted as the endogenous sponge of miRNA-21-3p, a miRNA belonging to the miRNA-21 family, which has been demonstrated to result in excessive proliferation of cholesteatoma keratinocytes [19]. Meanwhile, hsa_circRNA-101458 was able to interact with miRNA-let-7a-3p, a miRNA belonging to the miRNA-let-7a family, the upregulation of which has been confirmed to suppress proliferation of keratinocytes in cholesteatoma [19]. Consequently, circRNAs might play an important role in the pathogenesis of middle ear cholesteatoma.

By analyzing the differentially expressed circRNA between cholesteatoma and normal skin, expressions of hsa_circRNA_104327 and hsa_circRNA_404655 in cholesteatoma were significantly increased, while the expression of hsa_circRNA_000319 was significantly decreased. Moreover, our results revealed that hsa_circRNA_000319 interacted with miRNA-4436b-5p, hsa_circRNA_104327 interacted with miRNA-1525p, and hsa_circRNA_404655 interacted with miRNA-3664-3p. Some studies have found that the decreased expression of miRNA-3664-3p in spinal cord glioblastoma (SC-GBM) may lead to an increase in the expression of its target gene growth diferentiation factor 15 (GDF15), which in turn increases the expression of p53 and promotes tumor progression [20]. However, so far, the role of miRNA-3664-3p in cholesteatoma disease is not clear. It is well known that although cholesteatoma is pathologically benign, it is locally invasive and leads to bone resorption. We speculate that hsa_circRNA_404655, as a sponge of miRNA-3664-3p, may play a potential regulatory role in the proliferation and local invasion of cholesteatoma keratinocytes and participate in the formation of cholesteatoma. Moreover, we also discovered several targeted mRNAs for hsa_circRNA_404655 and miRNA-3664-3p, including IQGAP3, ROR2, EPHB1, NPM1, RCC2, NCOA5, and so on. Although these targeted mRNAs have not been investigated in middle ear cholesteatoma, their biological functions were explored in other proliferative 
and invasive diseases. For instance, IQGAP3, EPHB1, ROR2 and RCC2 were demonstrated to markedly enhance cell migration, invasion, and epithelial-to-mesenchymal transition in hepatocellular carcinoma, medulloblastoma and breast carcinoma, respectively [21-24]. Meanwhile, epithelial-to-mesenchymal transition was also observed in middle ear cholesteatoma [25]. Moreover, NPM1 contributed to enhanced cell proliferation with phosphatase and tensin homologue deleted on chromosome ten (PTEN) inactivation [26], while NCOA5 promotes proliferation, migration and invasion of colorectal cancer cells via activation of PI3K/Akt pathway [27]. Similarly, PTEN inactivation and PI3K/Akt pathway activation were also noticed in middle ear cholesteatoma [28]. These results provide new insights into the pathogenesis of cholesteatoma and suggest that circRNAs may be a potential target for the treatment of cholesteatoma. The specific mechanism and regulatory pathway need to be further studied. In addition, our study also found that the expression of hsa_circRNA_000319 was significantly decreased in cholesteatoma tissues, and caused the up-regulation of miR-4436b-5p expression in cholesteatoma tissues. However, its potential role needs to be further explored.

Through KEGG pathway analysis, autophagy and hypoxia inducible factor-1 (HIF-1) signaling pathway were indentified. Li et al [29]. proposed that enhanced autophagy might contribute to the pathogenesis of middle ear cholesteatoma through PI3K/Akt pathway activation. However, Ho ea al [30]. put forward that autophagy is significantly suppressed in cholesteatoma. Consequently, autophagy is involved in the pathogenesis of middle ear cholesteatoma with unclear function. Moreover, activated HIF-1 pathway was detected in middle ear cholesteatoma, indicating that cholesteatoma might be hypoxic [31]. Furthermore, KEGG pathway analysis exhibited several potential signaling pathway, which broaden our horizons on the pathogenesis of middle ear cholesteatoma.

More and more evidence shows that m6A modification can regulate the function of circRNAs. It is reported that m6A modification can also initiate circRNAs translation, and this m6A-driven translation requires the initiation factor eukaryotic translation initiation factor 4 gamma 2 (elF4G2) and m6A reader YTH domain family 3 (YTHDF3), which is enhanced by methyltransferase like 3 (METTL3) or METTL14, inhibited by demethylase fat mass and obesity associated protein (FTO), and upregulated upon heat shock [32]. Park et al [33]. demonstrated that m6A modification could reduce the stability of circRNA. In addition, m6A modification can also regulate cytoplasmic output. It has been found that YTH domaincontaining protein 1 (YTHDC1) mediates the nuclear output of circNSUN2 in a m6A methylationdependent manner, which increases the cytoplasmic expression of CircNSUN2 and promotes liver metastasis of colorectal cancer [34]. Some studies have found that unmodified circRNA is an effective adjuvant to induce specific $T$ and $B$ cell responses, and can activate Retinoic acid-inducible gene I (RIG-I) and innate immune signals with K63-polyubiquitin, while m6A RNA modification marks its own circRNA, and makes circRNA immune ineffective [35]. However, these potentially important functions of circRNA have not been fully studied. Therefore, the prediction and analysis of m6A modification of circRNA need more in-depth research.

\section{Conclusion}


In conclusion, in this study, totally 93 up-regulated and 85 down-regulated circRNAs were identified in midln conclusion, in this study, totally 93 up-regulated and 85 down-regulated circRNAs were demonstrated in middle ear cholesteatoma tissues. Compared with normal postauricular skin, the qRTPCR validation for dissimilarly expressed circRNAs identified that hsa_circRNA_104327 and hsa_circRNA_404655 were expressed significantly higher while hsa_circRNA_000319 expression significantly decreased in cholesteatoma tissue, suggesting that circRNAs may contribute to the pathogenesis of middle ear cholesteatoma. The exact mechanism needs to be further investigated. Targeting on theses circRNAs may be a candidate for new strategies in middle ear cholesteatoma therapy.

\section{Materials And Methods Patients and samples}

Acquired middle ear cholesteatoma tissues were obtained from patients undergoing cholesteatoma surgery between August 2019 and December 2019. Meanwhile, normal post-auricular skin samples were collected to be recognized as controls. All samples were stored in liquid nitrogen at once for circRNA array and qRT-PCR validation.

\section{RNA extraction}

Total RNAs were extracted using TRIzol reagent (Invitrogen; USA) according to the manufacturer's instructions. Then, RNasey mini kit (QIAGEN, German) was used to purify RNAs. Meanwhile, RNA concentrations were determined through NanoDrop ND-1000 instrument (Thermo, USA), and RNA integrity was assessed through gel electrophoresis.

\section{CircRNA labeling and microarray hybridization}

The sample preparation and circRNA microarray hybridization were performed based on the Arraystar's standard protocols. Briefly, total RNAs were digested with Rnase R (Epicentre, Inc.) to remove linear RNAs and enrich circular RNAs. Then, the enriched circular RNAs were amplified and transcribed into fluorescent cRNA utilizing a random priming method (Arraystar Super RNA Labeling Kit; Arraystar). The labeled cRNAs were hybridized onto the Arraystar Human circRNA Array V2 (8x15K, Arraystar), and incubated for 17 hours at $65^{\circ} \mathrm{C}$ in an Agilent Hybridization Oven. After having washed the slides, the arrays were scanned by the Agilent Scanner G2505C.

\section{Image acquisition and microarray analysis of circRNAs}


Agilent Feature Extraction software (version 11.0.1.1) was used to analyze acquired array images. Quantile normalization and subsequent data processing were performed using the R software limma package. Differentially expressed circRNAs with statistical significance between middle ear cholesteatoma and normal skin were identified using FC (fold change) cutoff or through volcano plot filtering, respectively. The value of $\mathrm{FC}^{3} 2$ and $\mathrm{P}<0.05$ was regarded as significantly differentially expressed. Hierarchical Clustering was performed to show the distinguishable circRNAs expression pattern between middle ear cholesteatoma and normal skin samples. Finally, the circRNA-miRNA interaction was predicted with Arraystar's home-made miRNA target prediction software based on TargetScan (http://www.targetscan.org) and miRanda (http://www.microrna.org).

\section{Validation for differentially expressed circRNAs by quantitative real-time PCR (qRT-PCR)}

Total cellular RNA from 10 middle ear cholesteatoma and 10 normal post-auricular skin tissues were used to validate circRNA expressions. Detail experimental operation was introduced in our previous study [15]. The specific amplification primer pairs were shown in Table 2.

Table 2. Primers for RT-qPCR validation 


\begin{tabular}{|ll|}
\hline Gene name & Primer sequences \\
\hline B-actin & F:5' GTGGCCGAGGACTTTGATTG3' \\
& R :5' CCTGTAACAACGCATCTCATATT3' \\
\hline hsa_circRNA_103670 & F:5' ATAACAATCTGTTACGGGTTTT 3' \\
& R:5' GGCATCCCTATTAGTCTTTCAA 3' \\
\hline hsa_circRNA_048764 & F:5' TGCCCTCTCCCTGAAATAAAG 3' \\
& R:5' GCAGTGCGGGAAACTTCTGT 3' \\
\hline hsa_CircRNA_404864 & F:5' TCTCCCAAGGAAGATACCGA 3' \\
& R:5' GCTCAGAGCAGTGCACATTATT 3' \\
\hline hsa_circRNA_104327 & F:5' TGTGTCAAATTTGTTCAAGACA 3' \\
& R:5' CAGGTTCTAGCAACTCACTTTG 3' \\
\hline hsa_circRNA_404655 & F:5' CAGCACTCCACAGCATCCACTA 3' \\
& R:5' TCCATTTCAATGGTAGCCTGCA 3' \\
\hline hsa_circRNA_101965 & F:5' GTCTTCCAGATCCACCAGGTT 3' \\
& R:5' TGCCATCTGTCAGAAACTTGAT 3' \\
\hline hsa_circRNA_100927 & F:5' GATAAAAGTGGATTGCAAGACT 3' \\
& R:5' TTCAAATAAACTGTCTGCCAAC 3' \\
\hline hsa_circRNA_000319 & F:5' TGTAGCTGTGGATCTACCAAAA 3' \\
& R:5' ACTTTGGGGCCTTTCAAGC 3' \\
\hline
\end{tabular}

circRNA, circulating RNA; F, forward; $R$, reverse.

\section{ceRNA network analysis}

Significantly differentially expressed circRNAs including hsa_circRNA_000319, hsa_circRNA_104327, and hsa_circRNA_404655 was selected to perform ceRNA network analysis. The cytoscape software V3.5.0 (San Diego, CA, USA) was adopted to construct circRNA-associated ceRNA network.

\section{Gene Ontology (GO) and Kyoto Encyclopedia Genes and Genomes (KEGG) pathway analysis}

GO classification (http://www.geneontology.org) and KEGG pathway analysis (http://www.genome.jp/kegg/) were applied to obtain utile information with respect to the functions of 
target genes in the pathogenesis of middle ear cholesteatoma. The GO classification provides a controlled vocabulary to describe gene and gene product attributes. The ontology covers three domains: biological process (BP), cellular component (CC) and molecular function (MF). Pathway analysis is a functional analysis mapping genes to KEGG pathways. A $P<0.05$ was considered to be statistically significant.

\section{Prediction of $\mathrm{N}^{6}$-methyladenosine $\left(\mathrm{m}^{6} \mathrm{~A}\right)$ modification on differentially expressed circRNAs}

Sequence-based RNA adenosine methylation site predictor (SRAMP) website (http://www.cuilab.cn/sramp) was searched in order to explore the m6A modification on differentially expressed circRNAs. Significantly differentially expressed circRNAs including hsa_circRNA_000319, hsa_circRNA_104327, and hsa_circRNA_404655 were selected to conduct the prediction of m6A modification sites on the RNA sequences of these 3 circRNAs.

\section{Abbreviations}

circRNA: circular RNA; miRNA: MicroRNA; mRNA: message RNA; MRE: miRNA response element; ceRNA: competing endogenous RNA; PCNA: proliferating cell nuclear antigen; CK: cytokeratin; qRT-PCR: quantitative real-time polymerase chain reaction; GO: Gene Ontology; KEGG: Kyoto Encyclopedia Genes and Genomes; BP: biological process; CC: cellular component; MF: molecular function; m6A:N6methyladenosine; IQGAP3: IQ motif-containing GTPase activating protein 3; ROR2: receptor tyrosine kinase-like orphan receptor 2; EPHB1: Ephrin Type-B Receptor 1; NPM1:nucleophosmin 1; RCC2: chromosome condensation 2; NCOA5: nuclear receptor coactivator 5; IGF2: insulin-like growth factor 2; PI3K: phosphatidylinositol 3-kinase; TIMP3: tissue inhibitor of matrix metalloproteinase 3; SC-GBM: spinal cord glioblastoma; GDF15: growth diferentiation factor 15; PTEN: phosphatase and tensin homologue deleted on chromosome ten; HIF-1: hypoxia inducible factor-1; elF4G2: eukaryotic translation initiation factor 4 gamma 2; YTHDF3: YTH domain family 3; METTL3: methyltransferase like 3; FTO: demethylase fat mass and obesity associated protein; YTHDC1: YTH domain-containing protein 1; RIG-I: Retinoic acid-inducible gene I.

\section{Declarations}

\section{Ethics approval and consent to participate}

This study procedure was approved by the Ethics Committee of Central South University, and written informed consent was acquired from all participants.

\section{Consent for publication}

Not applicable. 
Availability of data and materials

All data generated and analyzed during this study are included in this published article, detail data have been uploaded in https://www.ncbi.nlm.nih.gov/geo/query/acc.cgi?acc=GSE171006.

\section{Competing interests}

The authors declare that they have no competing interests.

\section{Funding}

This work was supported by the National Natural Science Foundation of China [grant number 82071036, grant number 82000973], Natural Science Foundation of Hunan Province [grant number 2019JJ50967区 2017JJ3441]

\section{Authors' contributions}

All authors read and approved the fnal manuscript

\section{Acknowledgements}

Not applicable.

\section{References}

1. Castle JT. Cholesteatoma Pearls: Practical Points and Update. Head Neck Pathol. 2018;12(3):41929.

2. Xie S, Wang X, Ren J, Liu W. The role of bone resorption in the etiopathogenesis of acquired middle ear cholesteatoma. Eur Arch Otorhinolaryngol. 2017;274(5):2071-8.

3. Bhutta MF, Williamson IG, Sudhoff HH. Cholesteatoma. BMJ. 2011;342:d1088.

4. Zhang ZG, Chen SJ, Sun W, Zheng YQ. Classification and surgical management of localized attic cholesteatoma: single-institution experience and follow-up. ORL J Otorhinolaryngol Relat Spec. 2010;72(2):96-100.

5. Jeck WR, Sharpless NE. Detecting and characterizing circular RNAs. Nat Biotechnol. 2014;32(5):45361.

6. Dai R, Ahmed SA. MicroRNA, a new paradigm for understanding immunoregulation, inflammation, and autoimmune diseases. Transl Res. 2011;157(4):163-79.

7. Hansen TB, Jensen TI, Clausen BH, Bramsen JB, Finsen B, Damgaard CK, et al. Natural RNA circles function as efficient microRNA sponges. Nature. 2013;495(7441):384-8.

8. Hansen TB, Kjems J, Damgaard CK. Circular RNA and miR-7 in cancer. Cancer Res. 2013;73(18):5609-12. 
9. Moldovan LI, Hansen TB, Venø MT, Okholm TLH, Andersen TL, Hager H, et al. High-throughput RNA sequencing from paired lesional- and non-lesional skin reveals major alterations in the psoriasis circRNAome. BMC Med Genomics. 2019;12(1):174.

10. Dang RY, Liu FL, Li Y. Circular RNA hsa_circ_0010729 regulates vascular endothelial cell proliferation and apoptosis by targeting the miR-186/HIF-1a axis. Biochem Biophys Res Commun. 2017;490(2):104-10.

11. Wang X, Cao X, Dong D, Shen X, Cheng J, Jiang R, et al. Circular RNA TTN Acts As a miR-432 Sponge to Facilitate Proliferation and Differentiation of Myoblasts via the IGF2/PI3K/AKT Signaling Pathway. Mol Ther Nucleic Acids. 2019;18:966-80.

12. Hamed MA, Nakata S, Shiogama K, Suzuki K, Sayed RH, Nishimura Y, et al. Cytokeratin 13, Cytokeratin 17, and Ki-67 Expression in Human Acquired Cholesteatoma and Their Correlation With Its Destructive Capacity. Clin Exp Otorhinolaryngol. 2017;10(3):213-20.

13. Liu W, Ren H, Ren J, Yin T, Hu B, Xie S, et al. The role of EGFR/PI3K/Akt/cyclinD1 signaling pathway in acquired middle ear cholesteatoma. Mediators Inflamm. 2013;2013:651207.

14. Liu W, Yin T, Ren J, Li L, Xiao Z, Chen X, et al. Activation of the EGFR/Akt/NF-kB/cyclinD1 survival signaling pathway in human cholesteatoma epithelium. Eur Arch Otorhinolaryngol. 2014;271(2):265-73.

15. Xie S, Liu X, Pan Z, Chen X, Peng A, Yin T, et al. Microarray Analysis of Differentially-expressed MicroRNAs in Acquired Middle Ear Cholesteatoma. Int J Med Sci. 2018;15(13):1547-54.

16. Pan G, Mao A, Liu J, Lu J, Ding J, Liu W. Circular RNA hsa_circ_0061825 (circ-TFF1) contributes to breast cancer progression through targeting miR-326/TFF1 signalling. Cell Prolif. 2020;53(2):e12720.

17. Yu J, Xu QG, Wang ZG, Yang Y, Zhang L, Ma JZ, et al. Circular RNA cSMARCA5 inhibits growth and metastasis in hepatocellular carcinoma. J Hepatol. 2018;68(6):1214-27.

18. Li F, Zhang L, Li W, Deng J, Zheng J, An M, et al. Circular RNA ITCH has inhibitory effect on ESCC by suppressing the Wnt/ $\beta$-catenin pathway. Oncotarget. 2015;6(8):6001-13.

19. Gao J, Tang Q, Xue R, Zhu X, Wang S, Zhang Y, et al. Comprehensive circular RNA expression profiling with associated ceRNA network reveals their therapeutic potential in cholesteatoma. Oncol Rep. 2020;43(4):1234-44.

20. An T, Fan T, Zhang XQ, Liu YF, Huang J, Liang C, et al. Comparison of Alterations in miRNA Expression in Matched Tissue and Blood Samples during Spinal Cord Glioma Progression [published correction appears in Sci Rep. 2020 Apr 22;10(1):6871]. Sci Rep. 2019;9(1):9169.

21. Shi Y, Qin N, Zhou Q, Chen Y, Huang S, Chen B, et al. Role of IQGAP3 in metastasis and epithelialmesenchymal transition in human hepatocellular carcinoma. J Transl Med. 2017;15(1):176.

22. Xu J, Shi J, Tang W, Jiang P, Guo M, Zhang B, et al. ROR2 promotes the epithelial-mesenchymal transition by regulating MAPK/p38 signaling pathway in breast cancer. J Cell Biochem. 2020;121(10):4142-53.

23. Chen Z, Wu W, Huang Y, Xie L, Li Y, Chen H, et al. RCC2 promotes breast cancer progression through regulation of Wnt signaling and inducing EMT. J Cancer. 2019;10(27):6837-47. 
24. Bhatia S, Baig NA, Timofeeva O, Pasquale EB, Hirsch K, MacDonald TJ, et al. Knockdown of EphB1 receptor decreases medulloblastoma cell growth and migration and increases cellular radiosensitization. Oncotarget. 2015;6(11):8929-46.

25. Takahashi M, Yamamoto-Fukuda T, Akiyama N, Motegi M, Yamamoto K, Tanaka Y, et al. Partial Epithelial-Mesenchymal Transition Was Observed Under p63 Expression in Acquired Middle Ear Cholesteatoma and Congenital Cholesteatoma. Otol Neurotol. 2019;40(8):e803-11.

26. Boudra R, Lagrafeuille R, Lours-Calet C, de Joussineau C, Loubeau-Legros G, Chaveroux C, et al. mTOR transcriptionally and post-transcriptionally regulates Npm1 gene expression to contribute to enhanced proliferation in cells with Pten inactivation. Cell Cycle. 2016;15(10):1352-62.

27. Sun $\mathrm{K}$, Wang S, He J, Xie Y, He Y, Wang Z, et al. NCOA5 promotes proliferation, migration and invasion of colorectal cancer cells via activation of PI3K/AKT pathway. Oncotarget. 2017;8(64):107932-46.

28. Liu W, Yin T, Ren J, Wang Y, Shen X, Li L, et al. Expression of PTEN, P-Akt and NF-KB in middle ear cholesteatoma epithelium and its significance. Chinese J Otol. 2011;9(2):206-11.

29. Li Q, Ao Y, Yu Q, Zhou S. Role of Autophagy in Acquired Cholesteatoma. Otol Neurotol. 2019;40(10):e993-8.

30. Ho KY, Huang CJ, Hung CC, Wu YR, Chiu CC, Chien CY, et al. Autophagy Is Deficient and May be Negatively Regulated by SERPINB3 in Middle Ear Cholesteatoma. Otol Neurotol. 2020;41(7):e881-8.

31. Adunka O, Gstoettner W, Knecht R, Kierner AC. Expression of hypoxia inducible factor 1 alpha and Von Hippel Lindau protein in human middle ear cholesteatoma. Laryngoscope. 2003;113(7):1210-5.

32. Yang $Y$, Fan $X$, Mao $M$, Song $X$, Wu P, Zhang $Y$, et al. Extensive translation of circular RNAs driven by N6-methyladenosine. Cell Res. 2017;27(5):626-41.

33. Park OH, Ha H, Lee Y, Boo SH, Kwon DH, Song HK, et al. Endoribonucleolytic Cleavage of m6AContaining RNAs by RNase P/MRP Complex. Mol Cell. 2019;74(3):494-507.e8.

34. Chen RX, Chen X, Xia LP, Zhang JX, Pan ZZ, Ma XD, et al. N6-methyladenosine modification of circNSUN2 facilitates cytoplasmic export and stabilizes HMGA2 to promote colorectal liver metastasis. Nat Commun. 2019;10(1):4695.

35. Chen YG, Chen R, Ahmad S, Verma R, Kasturi SP, Amaya L, et al. N6-Methyladenosine Modification Controls Circular RNA Immunity. Mol Cell. 2019;76(1):96-109.e9.

\section{Figures}



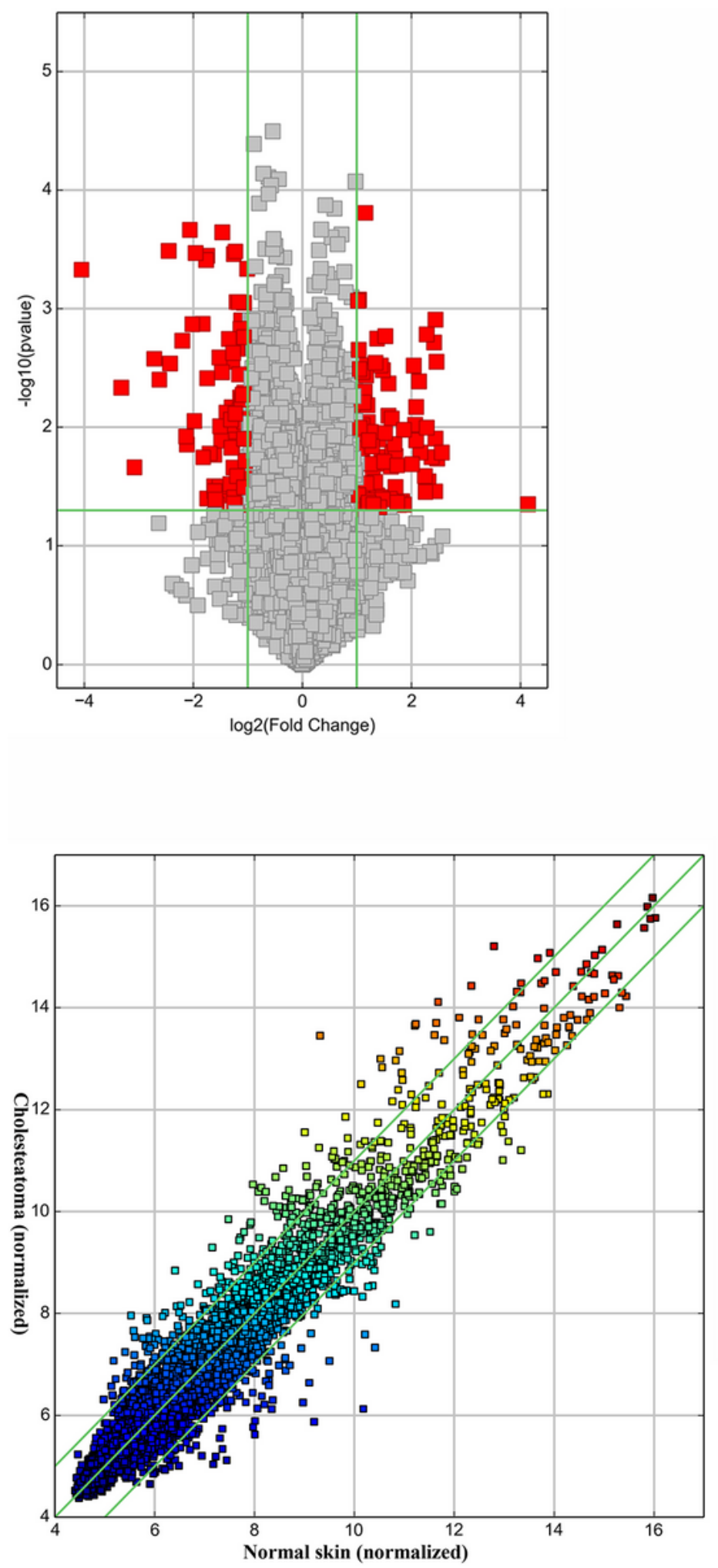
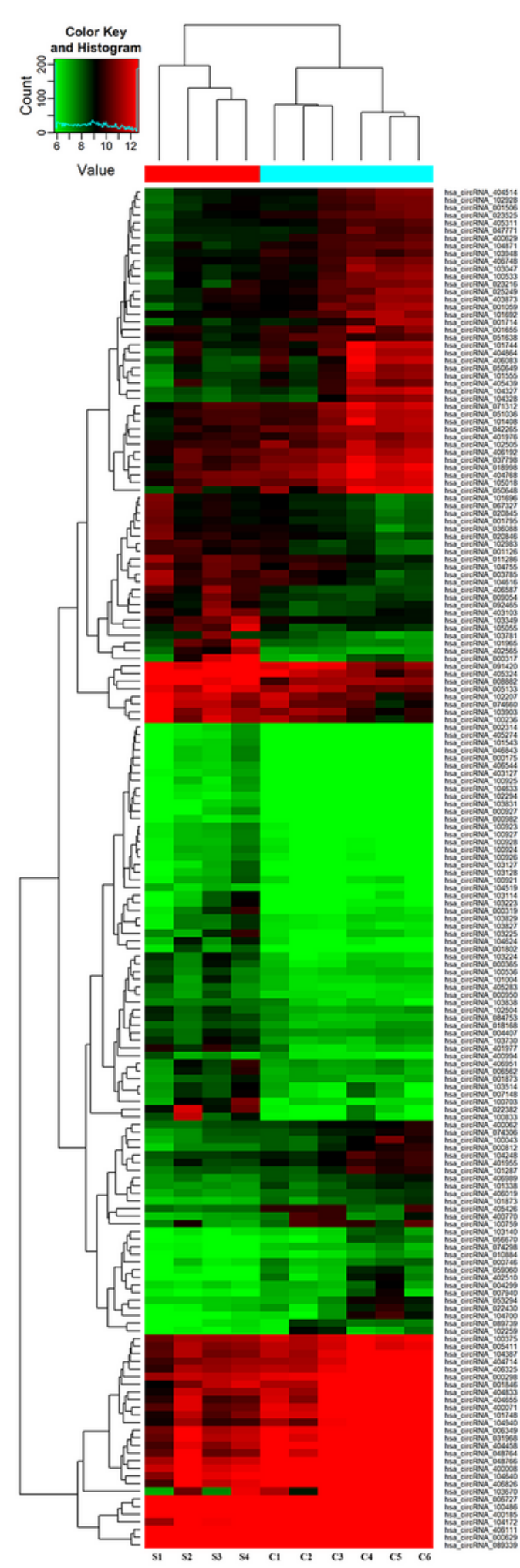

\section{Figure 1}

The differentially expressed circRNAs were displayed in a volcano plot $(1 A)$, and then subjected to a scatter plot (1B) and hierarchical clustering analysis (1C), to display the variation and differentiation between middle ear cholesteatoma and normal kin. Moreover, among these 178 significantly differentially expressed circRNAs, a total of 145 were exonic. 

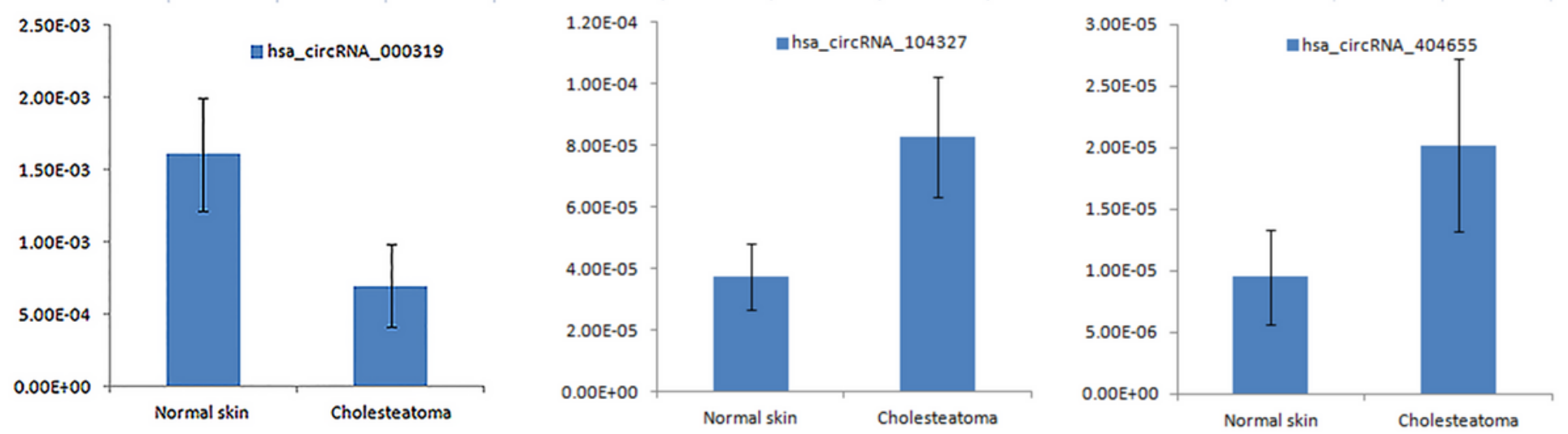

Figure 2

The validation result was accordance with the microarray discoveries partly: compared with normal skin, expressions of hsa_circRNA_104327 and hsa_circRNA_404655 were significantly higher in middle ear cholesteatoma $(P<0.05)$, while the expression of hsa_circRNA_000319 was significantly decreased $(P<0.05)$

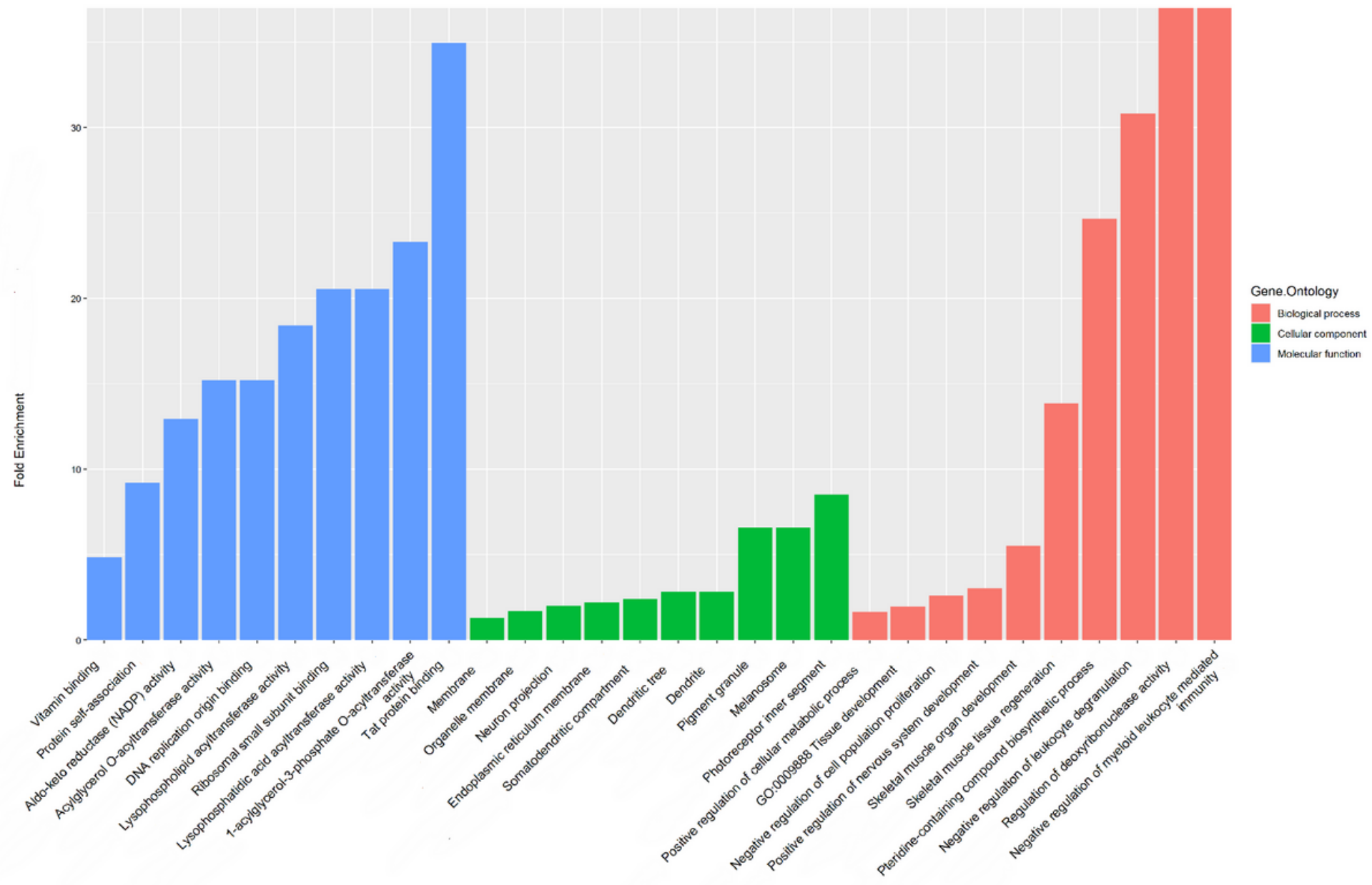

\section{Figure 3}

The top-10 enriched BP, CC, and MF domains in GO classification were displayed in Figure. 
Ubiquinone and other terpenoid-quinone biosynthesis

One carbon pool by folate

Fluid shear stress and atherosclerosis

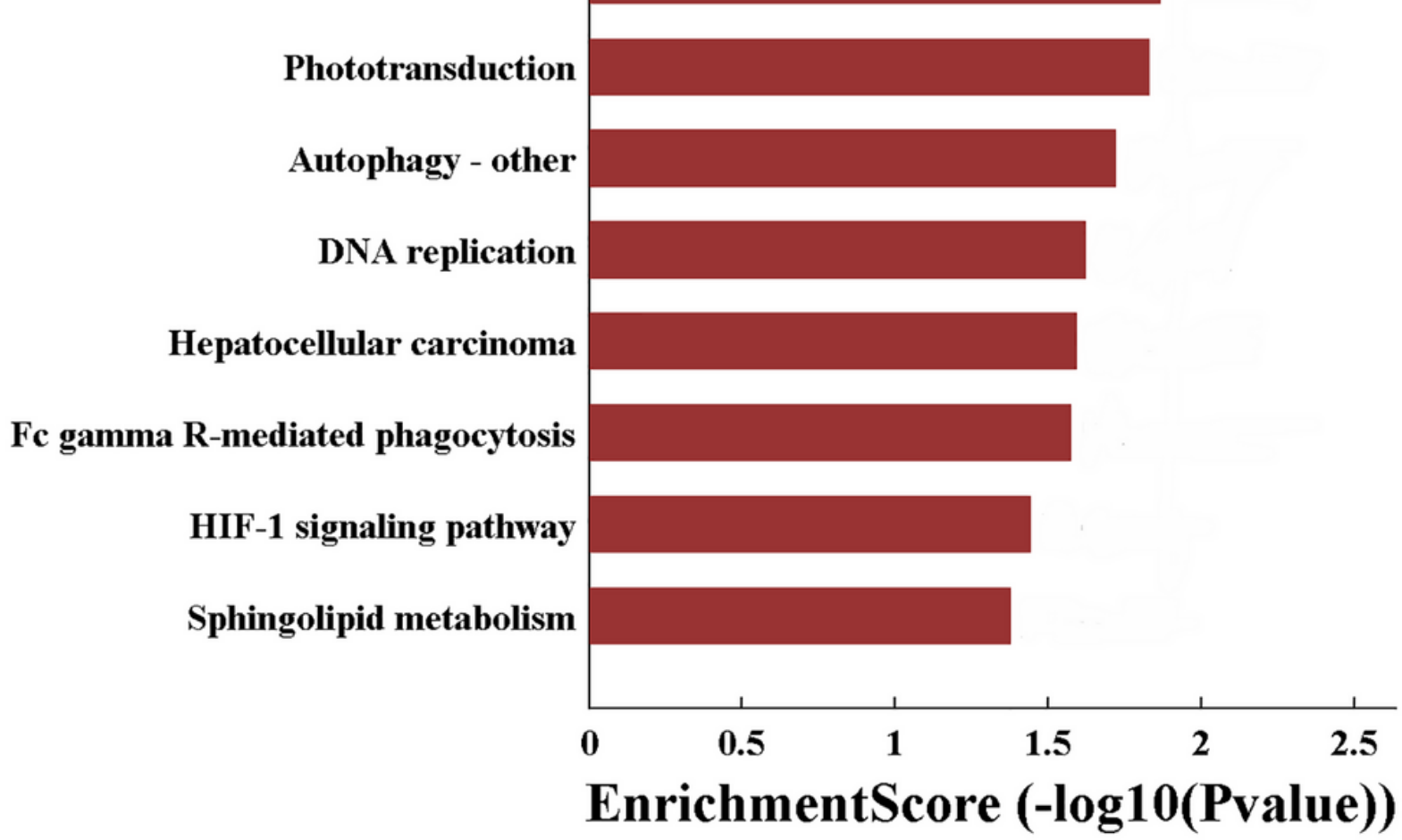

Figure 4

Meanwhile, regarding on KEGG pathway analysis, 11 signaling pathways in total were demonstrated to be enriched. 


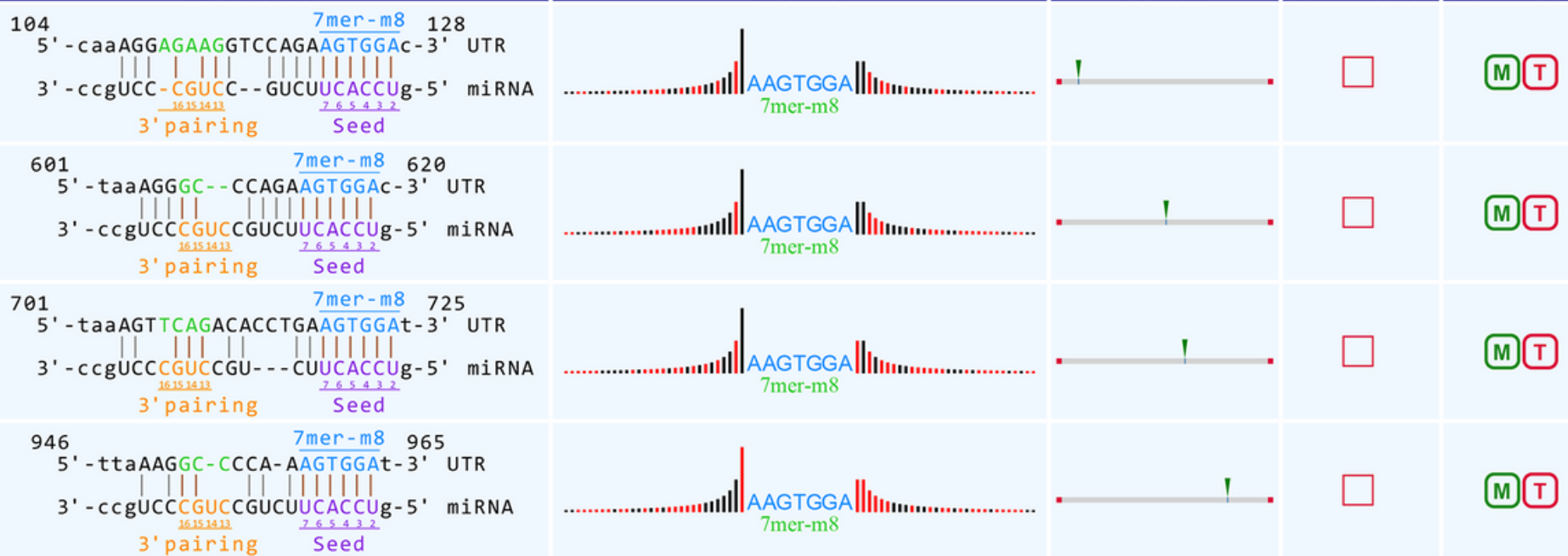

M T

\section{D Structure}

91 ' - ccacaaAGTGAGTTGCTAGAACC $\mathrm{t}-3^{\prime}$ UTR

3' - ucagccUCACAUAGU-GUCUUGGa-5' miRnA

3'pairing $\frac{765432}{\text { Seed }}$

$$
\text { offset }
$$

\section{D Structure}

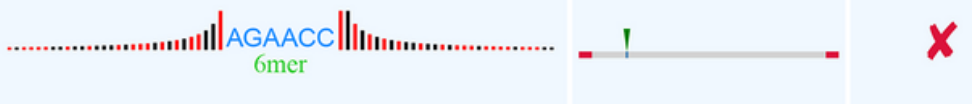
6 mer
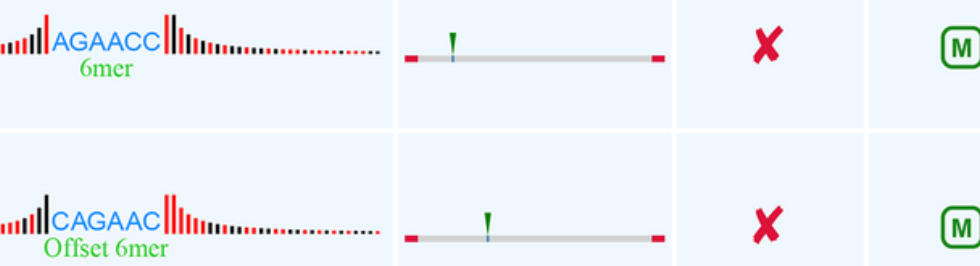

(M)

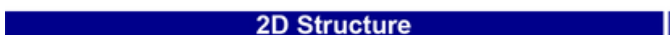

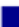
Local AU
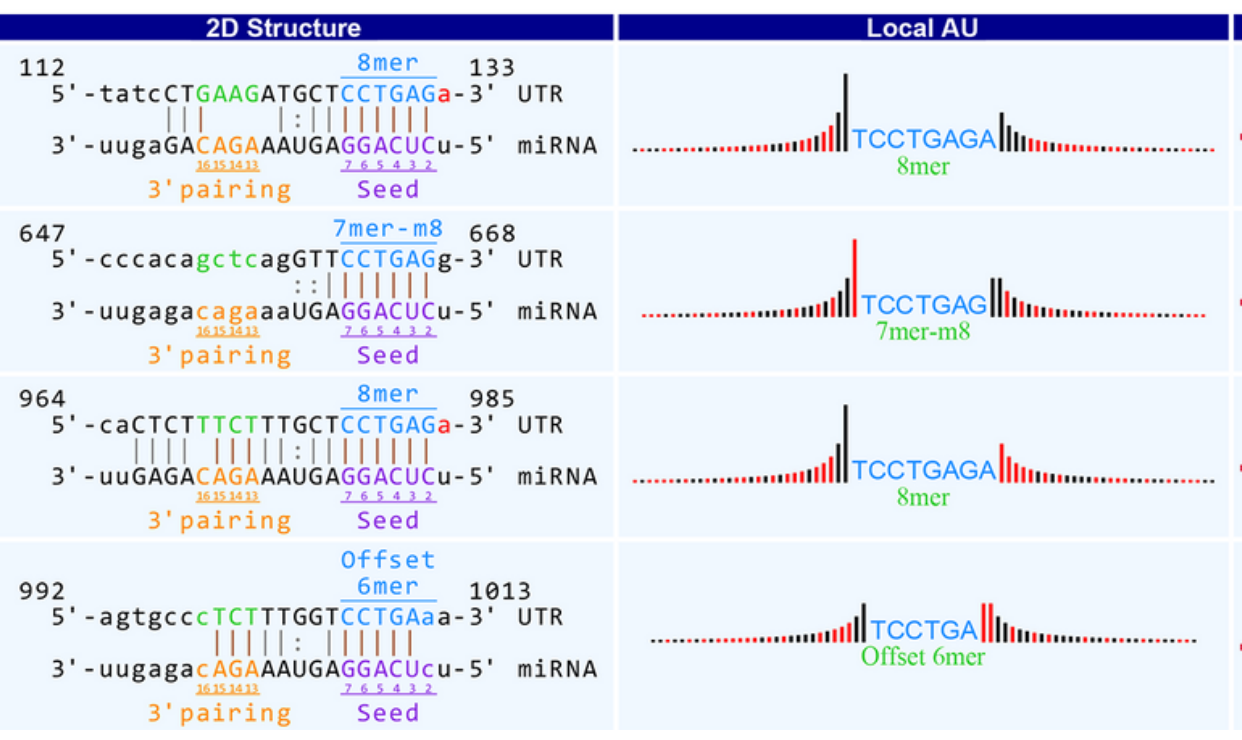

$\left.\right|_{\mathrm{TC}}$ CCTGAG |lliı.ı.

IIItcctgagal|ı.ı. 8 mer
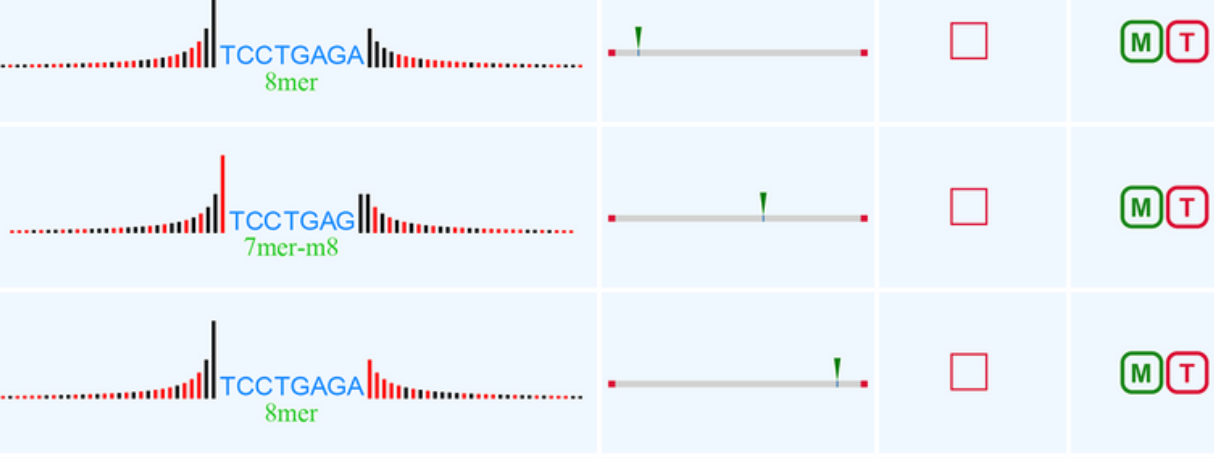
I| TCCTGA |lııı Offset $6 \mathrm{mer}$

\section{Figure 5}

We discovered that hsa_circRNA_000319 interacted with hsa-miRNA-4436b-5p, hsa_circRNA_104327 interacted with miRNA-152-5p, and hsa_circRNA_404655 interacted with miRNA-3664-3p, with a complementarity based on 7 mer-m8, 6 mer, offset 6 mer, or 8 mer matching types. 


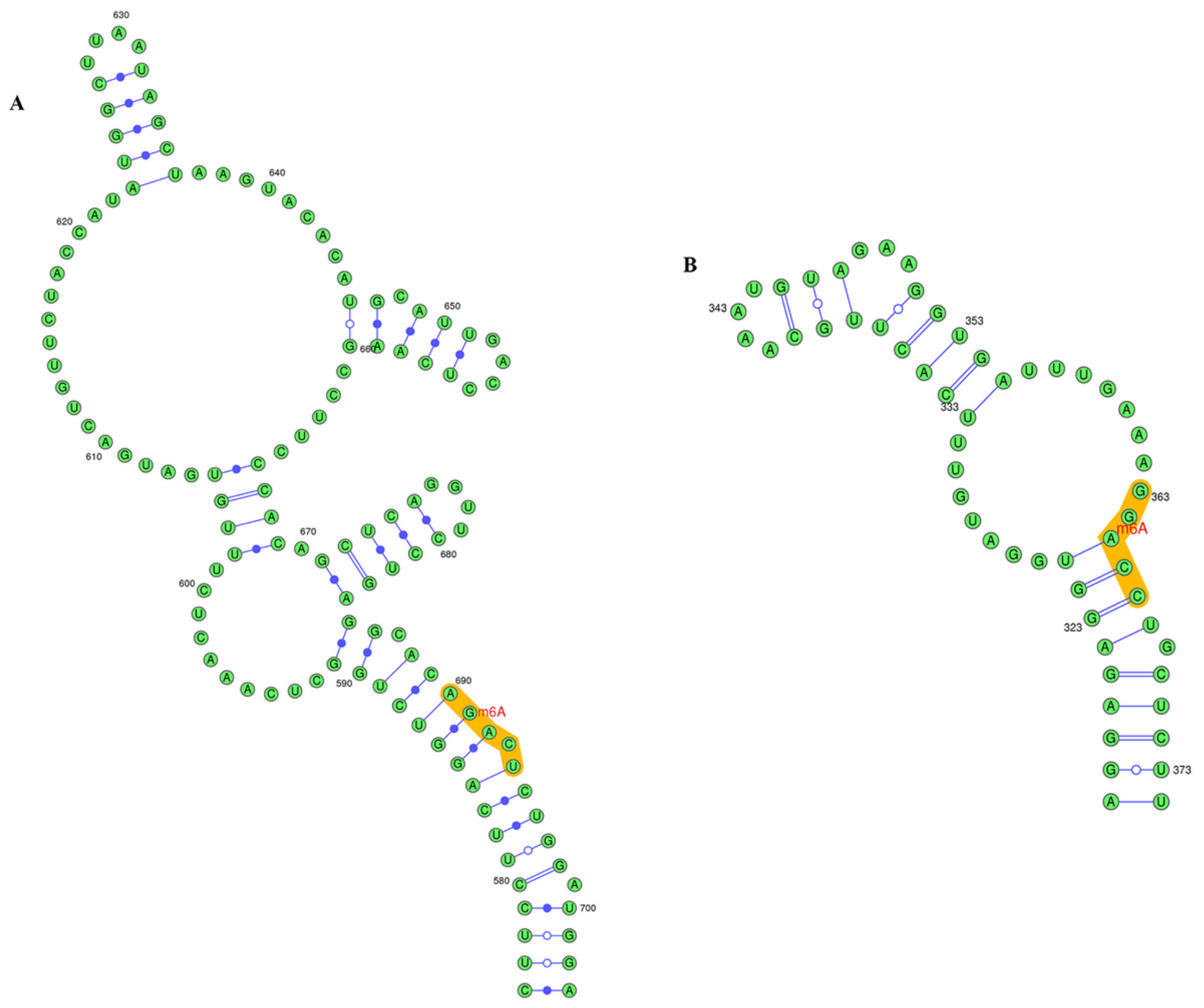

\section{Figure 6}

In our study, we discovered that hsa_circRNA_000319 possessed $4 \mathrm{m6A}$ sites with very high confidence in total, and hsa_circRNA_404655 had 3 m6A sites with high confidence, while no m6A site with very high or high confidence was found for hsa_circRNA_104327. Details were displayed in Table 1 and Figure.

\section{Supplementary Files}

This is a list of supplementary files associated with this preprint. Click to download.

- Figs1.tif 